\title{
Solution of the Oldano-Barbero paradox
}

\author{
S. Stallinga and G. Vertogen \\ Institute for Theoretical Physics and Research Institute for Materials, University of Nijmegen, Toernooiveld, \\ 6525 ED Nijmegen, The Netherlands \\ (Received 20 January 1995)
}

\begin{abstract}
The appearance of the splay-bend elastic constant $K_{13}$ in the theory of elasticity of nematic liquid crystals causes a serious problem. This problem is known as the Oldano-Barbero paradox. The approaches of Barbero et al., Faetti, and Pergamenshchik for solving this paradox are analyzed in view of two starting points of the continuum theory. The analysis clearly shows the imperfections of their treatments. In order to remedy these imperfections a proposal is made to eliminate the paradox in accordance with the assumptions of the continuum theory. The solution requires a modification of the surface free energy density, namely, the derivatives of the director field normal to the surface must be excluded.
\end{abstract}

PACS number(s): 61.30.-v, 03.40.Dz

\section{INTRODUCTION}

The influence of the surface on the equilibrium director field of a liquid crystal is represented by the boundary conditions. There are two kinds of boundary conditions, one kind pertaining to strong anchoring of the liquid crystal at the surface, and the other kind to weak anchoring of the liquid crystal at the surface. The boundary conditions for strong anchoring are fixed, i.e., the director field is assumed to be fixed along a certain preferential direction. The boundary conditions for weak anchoring, on the other hand, follow from the minimization of the total free energy, which is the sum of a bulk part and a surface part. Thus weak anchoring involves the contribution of surface elasticity to the surface free energy. A serious problem, however, arises with the incorporation of surface elastic terms, notably the splay-bend term. Then the continuum theory appears to lead to a discontinuity of the director field at the boundaries. This result is known as the Oldano-Barbero paradox (OB paradox) [1].

Two ways of approach have been proposed for solving this paradox. The first is due to Barbero et al. $[2,3]$ and Faetti $[4,5]$ and the second to Pergamenshchik [6]. Neither approach turns out to be entirely satisfactory. The first allows for a large variation of the director field in a microscopically thin region near the boundary and thus violates a starting point of the continuum theory. The second one does not describe an equilibrium situation, as the total torque is unequal to zero.

The aim of the present paper is to solve the OB paradox by combining the attractive features of the existing treatments and removing their unattractive ones. The paper is organized in the following way. In Sec. II the necessary conditions are discussed for the validity of the approximated elastic free energy. The OB paradox is discussed in Sec. III. The two mentioned solutions are analyzed in Secs. IV and V, followed by a discussion in Sec. VI. Finally Sec. VII contains the main conclusions.

\section{CONDITIONS FOR THE VALIDITY OF THE APPROXIMATED ELASTIC FREE ENERGY}

The elastic contribution to the free energy $F_{\mathrm{el}}$ is defined as the increase of the free energy due to the spatial variations of the director field:

$$
F_{\mathrm{el}}=\int d V f_{d}
$$

where $f_{d}$ is the deformation free energy density, which can be expressed as a series expansion in the spatial derivatives of the director field $\boldsymbol{n}(\boldsymbol{r})$. Usually $f_{d}$ is approximated by an expression which only contains terms with low-order spatial derivatives. Consequently $F_{\text {el }}$ can be written as

$$
F_{\text {el }}=F_{\text {app }}+R,
$$

where $F_{\text {app }}$ is the approximate elastic free energy based upon the approximated $f_{d}$ and $R$ is the result of the remaining higher-order terms. The approximation is valid provided that

$$
\left|F_{\text {app }}\right| \gg|R| .
$$

This means that two necessary conditions must be satisfied.

(i) The deformation of the director field must be small, or mathematically

$$
L \gg a,
$$

where $L$ is the smallest wavelength of the relevant Fourier components of the director field and $a$ the average intermolecular distance. Condition (4) is necessary for the validity of (3), as can be seen by estimating the order of magnitude of the different terms. Consider to that end a term in $f_{d}$ containing $k$ spatial derivatives. Each spatial derivative $\partial_{\alpha}$ introduces a factor $L^{-1}$. The $k$ th 
order elastic constant can be expected to be of the order $U a^{k}$, where $U$ is of the order of an intermolecular interaction energy divided by the average volume per molecule. Then the considered term is of the order $U(a / L)^{k}$, implying that the higher-order terms cannot be neglected if $L \sim a$.

(ii) The approximated elastic free energy must have a minimum, i.e., there must be a finite energy $F_{\min }$ such that

$$
F_{\text {app }} \geq F_{\min } \text {. }
$$

This condition follows directly from the apparent stability of nematic liquid crystals.

\section{THE OLDANO-BARBERO PARADOX}

The usual approximation of the elastic free energy density consists of all terms containing two spatial derivatives. The deformation free energy density can then be expressed as the sum of the Frank free energy density [7]

$$
\begin{aligned}
f_{b}= & \frac{1}{2} K_{11}(\nabla \cdot n)^{2}+\frac{1}{2} K_{22}[\boldsymbol{n} \cdot(\nabla \times n)]^{2} \\
& +\frac{1}{2} K_{33}[\boldsymbol{n} \times(\boldsymbol{\nabla} \times \boldsymbol{n})]^{2},
\end{aligned}
$$

and the additional divergence terms [8]

$$
\begin{aligned}
\partial_{\alpha} f_{\mathrm{s} \alpha}= & \frac{1}{2}\left(K_{22}+K_{24}\right) \nabla \cdot[(\boldsymbol{n} \cdot \boldsymbol{\nabla}) \boldsymbol{n}-\boldsymbol{n}(\boldsymbol{\nabla} \cdot \boldsymbol{n})] \\
& +K_{13} \boldsymbol{\nabla} \cdot[\boldsymbol{n}(\boldsymbol{\nabla} \cdot \boldsymbol{n})] .
\end{aligned}
$$

Here $K_{11}, K_{22}, K_{33}, K_{24}$, and $K_{13}$ are the elastic constants for splay, twist, bend, saddle splay and splay bend, respectively. According to Gauss's theorem the divergence terms only contribute to the surface free energy density. Therefore these terms are called surface terms. The elastic free energy can now be written as the sum of a bulk part, containing the three Frank terms, and a surface part, containing the two new terms:

$$
F_{\mathrm{el}}=\int d V f_{b}+\int d S f_{s}
$$

with

$$
f_{s}=p_{\alpha} f_{s \alpha}
$$

where $\boldsymbol{p}$ is a unit vector perpendicular to the surface, pointing outwards.

An important step for solving the OB paradox appears to be a decomposition of the surface free energy density $f_{s}$ in two terms. The first term $f_{s \|}$ relates to the derivatives of the director field that are tangential to the surface, whereas the second term $f_{s \perp}$ only concerns the derivatives normal to the surface. Thus

$$
f_{s}=f_{s \|}+f_{s \perp}
$$

where $f_{s \|}$ only depends on $t_{1 \beta} \partial_{\beta} n_{\alpha}$ and $t_{2 \beta} \partial_{\beta} n_{\alpha}$, with $t_{1}$ and $t_{2}$ two orthonormal vectors tangential to the surface:

$$
\begin{aligned}
f_{s \|}= & \frac{1}{2}\left(K_{22}+K_{24}\right) \boldsymbol{p} \cdot[(\boldsymbol{n} \cdot \boldsymbol{\nabla}) \boldsymbol{n}-\boldsymbol{n}(\boldsymbol{\nabla} \cdot \boldsymbol{n})] \\
& +K_{13}(\boldsymbol{p} \cdot \boldsymbol{n})[\boldsymbol{\nabla} \cdot \boldsymbol{n}-\boldsymbol{p} \cdot(\boldsymbol{p} \cdot \boldsymbol{\nabla}) \boldsymbol{n}] \\
= & \frac{1}{2}\left(K_{22}+K_{24}\right) \sum_{i=1,2}\left(t_{i \alpha} p_{\gamma}-p_{\alpha} t_{i \gamma}\right) n_{\alpha} t_{i \beta} \partial_{\beta} n_{\gamma} \\
& +K_{13} \sum_{i=1,2} n_{\alpha} p_{\alpha} t_{i \beta} t_{i \gamma} \partial_{\beta} n_{\gamma}
\end{aligned}
$$

and $f_{s \perp}$ only depends on $p_{\beta} \partial_{\beta} n_{\alpha}$ :

$$
\begin{aligned}
f_{s \perp} & =K_{13}(\boldsymbol{p} \cdot \boldsymbol{n}) \boldsymbol{p} \cdot(\boldsymbol{p} \cdot \nabla) \boldsymbol{n} \\
& =K_{13} n_{\alpha} p_{\alpha} p_{\beta} p_{\gamma} \partial_{\beta} n_{\gamma} .
\end{aligned}
$$

As to the contribution due to externally applied fields, only a magnetic field will be considered. The magnetic contribution to the free energy density can be written as

$$
F_{\mathrm{mag}}=\int d V f_{m}
$$

with the magnetic free energy density $f_{m}$ given by

$$
f_{m}=-\frac{1}{2} \frac{\Delta \chi}{\mu_{0}}(\boldsymbol{B} \cdot \boldsymbol{n})^{2},
$$

where $\Delta \chi$ is the magnetic anisotropy, that is assumed to be positive for reasons of convenience, $\mu_{0}$ the permeability in vacuum, and $\boldsymbol{B}$ the magnetic field.

The contribution to the free energy due to the interaction of the liquid crystal with the surrounding material is called the anchoring energy. It can be expressed as the surface integral

$$
F_{\text {anch }}=\int d S \sigma,
$$

where the anchoring free energy density is usually supposed to be of the Rapini-Papoular form [9]:

$$
\sigma=-\frac{1}{2} C(\boldsymbol{\Pi} \cdot \boldsymbol{n})^{2}
$$

with $\Pi$ a unit vector along the so-called easy direction and $C$ the anchoring constant.

The total free energy can thus be expressed as

$$
\begin{aligned}
F & =F_{\mathrm{el}}+F_{\mathrm{mag}}+F_{\text {anch }} \\
& =\int d V\left(f_{b}+f_{m}\right)+\int d S\left(\sigma+f_{s}\right) .
\end{aligned}
$$

Strong anchoring can be described in the limit of an infinite anchoring constant $C$. Then the equilibrium director field can be found by minimizing the bulk part of (16) under the constraint that $\boldsymbol{n}= \pm \boldsymbol{\Pi}$ at the boundary.

In order to determine the equilibrium director field in the general case of weak anchoring, the total free energy (16) must be minimized using a variational procedure. The director field is parametrized by two angles, the tilt angle $\theta(\boldsymbol{r})$ and the twist angle $\phi(\boldsymbol{r})$ :

$$
\boldsymbol{n}(\boldsymbol{r})=(\sin \theta(\boldsymbol{r}) \cos \phi(\boldsymbol{r}), \sin \theta(\boldsymbol{r}) \sin \phi(\boldsymbol{r}), \cos \theta(\boldsymbol{r})) .
$$


In the case of strong anchoring only variations $\delta \theta$ and $\delta \phi$ that vanish at the boundary need to be considered, as the director field at the boundary is assumed to be fixed along the easy direction. In the case of weak anchoring, on the other hand, more general variations $\delta \theta$ and $\delta \phi$ must be considered. Now variations at the boundaries must be taken into account as well. The variation in the total free energy can thus be expressed as

$$
\begin{aligned}
\delta F= & \sum_{\chi=\theta, \phi} \int d V\left[\left(\frac{\partial f_{m}}{\partial \chi}+\frac{\partial f_{b}}{\partial \chi}\right) \delta \chi+\left(\frac{\partial f_{b}}{\partial \partial_{\beta} \chi}\right) \delta \partial_{\beta} \chi\right] \\
& +\sum_{\chi=\theta, \phi} \int d S\left[\left(\frac{\partial \sigma}{\partial \chi}+\frac{\partial f_{s}}{\partial \chi}\right) \delta \chi+\left(\frac{\partial f_{s}}{\partial \partial_{\beta} \chi}\right) \delta \partial_{\beta} \chi\right] .
\end{aligned}
$$

Using Gauss's theorem it follows that

$$
\begin{aligned}
\int d V\left(\frac{\partial f_{b}}{\partial \partial_{\beta} \chi}\right) \delta \partial_{\beta} \chi= & \int d V \partial_{\beta}\left(\frac{\partial f_{b}}{\partial \partial_{\beta} \chi} \delta \chi\right) \\
& -\int d V \partial_{\beta}\left(\frac{\partial f_{b}}{\partial \partial_{\beta} \chi}\right) \delta \chi \\
= & \int d S p_{\beta} \frac{\partial f_{b}}{\partial \partial_{\beta} \chi} \delta \chi \\
& -\int d V \partial_{\beta}\left(\frac{\partial f_{b}}{\partial \partial_{\beta} \chi}\right) \delta \chi
\end{aligned}
$$

Then the variation in the free energy can be written as

$$
\begin{aligned}
\delta F= & \sum_{\chi=\theta, \phi} \int d V\left[\frac{\partial f_{m}}{\partial \chi}+\frac{\partial f_{b}}{\partial \chi}-\partial_{\beta}\left(\frac{\partial f_{b}}{\partial \partial_{\beta} \chi}\right)\right] \delta \chi \\
& +\sum_{\chi=\theta, \phi} \int d S\left[\frac{\partial \sigma}{\partial \chi}+p_{\beta} \frac{\partial f_{b}}{\partial \partial_{\beta} \chi}+\frac{\partial f_{s}}{\partial \chi}\right] \delta \chi \\
& +\sum_{\chi=\theta, \phi} \int d S\left[\frac{\partial f_{s}}{\partial \partial_{\beta} \chi}\right] \delta \partial_{\beta} \chi .
\end{aligned}
$$

Next it is important to realize that the last term on the right hand side of (20) can be partly incorporated into the other surface term on the right hand side of (20). To that end the two Gaussian coordinates $q_{1}$ and $q_{2}$, that label all points on the surface, are introduced. The dependence of the surface coordinates on the Gaussian coordinates, $x_{\alpha}\left(q_{1}, q_{2}\right)$, is chosen such that the two orthonormal vectors $t_{1}$ and $t_{2}$ can be expressed as

$$
t_{i \alpha}=\frac{\partial x_{\alpha}}{\partial q_{i}}, \quad \text { with } i=1,2
$$

Using the chain rule

$$
\frac{\partial}{\partial q_{i}}=t_{i \alpha} \partial_{\alpha}
$$

and the expression for the Kronecker tensor

$$
\delta_{\alpha \beta}=\sum_{i=1,2} t_{i \alpha} t_{i \beta}+p_{\alpha} p_{\beta}
$$

which follows from the orthonormality of $t_{1}, t_{2}$, and $p$, it follows that

$$
\begin{aligned}
\int d S & \frac{\partial f_{s}}{\partial \partial_{\beta} \chi} \delta \partial_{\beta} \chi \\
& =\int d S \frac{\partial f_{s}}{\partial \partial_{\beta} \chi}\left(\sum_{i=1,2} t_{i \beta} t_{i \mu}+p_{\beta} p_{\mu}\right) \delta \partial_{\mu} \chi \\
& =\int d S \sum_{i=1,2} t_{i \beta} \frac{\partial f_{s \|}}{\partial \partial_{\beta} \chi} \delta \frac{\partial \chi}{\partial q_{i}}+\int d S p_{\beta} \frac{\partial f_{s \perp}}{\partial \partial_{\beta} \chi} \delta p_{\mu} \partial_{\mu} \chi
\end{aligned}
$$

for $\chi=\theta, \phi$.

Now the relevance of the decomposition (10) becomes clear. Namely, the first term on the right hand side of (24) can be incorporated into the second term on the right hand side of (20) by means of a partial integration, whereas the second term cannot. Using

$$
d S=J\left(q_{1}, q_{2}\right) d q_{1} d q_{2},
$$

where $J\left(q_{1}, q_{2}\right)$ is the Jacobian, and assuming that the surface is closed or that $\delta \theta$ and $\delta \phi$ vanish at infinity in case the surface extends to infinity, it follows that

$$
\begin{aligned}
\int d S \sum_{i=1,2} t_{i \beta} \frac{\partial f_{s \|}}{\partial \partial_{\beta} \chi} \delta \frac{\partial \chi}{\partial q_{i}} & =\int d q_{1} d q_{2} \sum_{i=1,2} J\left(q_{1}, q_{2}\right) t_{i \beta} \frac{\partial f_{s \|}}{\partial \partial_{\beta} \chi} \frac{\partial}{\partial q_{i}}(\delta \chi) \\
& =-\int d q_{1} d q_{2} \sum_{i=1,2} \frac{\partial}{\partial q_{i}}\left[J\left(q_{1}, q_{2}\right) t_{i \beta} \frac{\partial f_{s \|}}{\partial \partial_{\beta} \chi}\right] \delta \chi \\
& =-\int d S \sum_{i=1,2} \frac{1}{J\left(q_{1}, q_{2}\right)} t_{i \mu} \partial_{\mu}\left[J\left(q_{1}, q_{2}\right) t_{i \beta} \frac{\partial f_{s \|}}{\partial \partial_{\beta} \chi}\right] \delta \chi
\end{aligned}
$$


for $\chi=\theta, \phi$. The variation in the total free energy (20) can thus be expressed as

$$
\begin{aligned}
\delta F= & \sum_{\chi=\theta, \phi} \int d V\left[\frac{\partial f_{m}}{\partial \chi}+\frac{\partial f_{b}}{\partial \chi}-\partial_{\beta}\left(\frac{\partial f_{b}}{\partial \partial_{\beta} \chi}\right)\right] \delta \chi \\
& +\sum_{\chi=\theta, \phi} \int d S\left\{\frac{\partial \sigma}{\partial \chi}+p_{\beta} \frac{\partial f_{b}}{\partial \partial_{\beta} \chi}+\frac{\partial f_{s}}{\partial \chi}\right. \\
& \left.-\sum_{i=1,2} \frac{1}{J\left(q_{1}, q_{2}\right)} t_{i \mu} \partial_{\mu}\left[J\left(q_{1}, q_{2}\right) t_{i \beta} \frac{\partial f_{s} \|}{\partial \partial_{\beta} \chi}\right]\right\} \delta \chi \\
& +\sum_{\chi=\theta, \phi} \int d S\left(p_{\beta} \frac{\partial f_{s \perp}}{\partial \partial_{\beta} \chi}\right) \delta p_{\mu} \partial_{\mu} \chi .
\end{aligned}
$$

Expressions for the variation in the total free energy similar to (27) can also be derived using tensor calculus in a general curvilinear coordinate frame [6] or using Stokes's theorem [10].

The third term on the right hand side of (27) is solely due to the appearance of the surface term of the splaybend type, i.e., it does not contain the elastic constant $K_{24}$ but only the elastic constant $K_{13}$, as can be seen from Eqs. (11). This result has also been derived in Refs. $[3,6]$. Clearly, the functional $F$ is extremal if the tilt and twist angles satisfy the Euler-Lagrange equations

$$
\partial_{\beta}\left(\frac{\partial f_{b}}{\partial \partial_{\beta} \chi}\right)-\frac{\partial f_{b}}{\partial \chi}-\frac{\partial f_{m}}{\partial \chi}=0
$$

for $\chi=\theta, \phi$, and the boundary conditions

$$
\begin{gathered}
\frac{\partial \sigma}{\partial \chi}+p_{\beta} \frac{\partial f_{b}}{\partial \partial_{\beta} \chi}+\frac{\partial f_{s}}{\partial \chi} \\
-\sum_{i=1,2} \frac{1}{J\left(q_{1}, q_{2}\right)} t_{i \mu} \partial_{\mu}\left[J\left(q_{1}, q_{2}\right) t_{i \beta} \frac{\partial f_{s \|}}{\partial \partial_{\beta} \chi}\right]=0 \\
p_{\beta} \frac{\partial f_{s \perp}}{\partial \partial_{\beta} \chi}=0
\end{gathered}
$$

for $\chi=\theta, \phi$. The second boundary condition, which is a consequence of taking into account the $K_{13}$ term, leads to the Oldano-Barbero paradox [1].

Consider, for example, the geometry with boundaries at $x=0$ and $x=d$, an easy axis $\Pi=\cos \theta_{p} \hat{e}_{z}+\sin \theta_{p} \hat{e}_{x}$, and a magnetic field $\boldsymbol{B}=B \hat{\boldsymbol{e}}_{\boldsymbol{x}}$. This geometry is called the splay-bend geometry. Assume, for the sake of simplicity, that $K_{11}=K_{33}=K$, and that the director field only depends on the tilt angle $\theta$, which itself only depends on the $x$ coordinate. Then the free energy is given by

$$
\begin{aligned}
F= & \int_{0}^{d} d x\left(\frac{1}{2} K \theta^{\prime 2}-\frac{1}{2} \frac{\Delta \chi B^{2}}{\mu_{0}} \sin ^{2} \theta\right) \\
& +\frac{1}{2} C \sin ^{2}\left[\theta(0)-\theta_{p}\right]+\frac{1}{2} C \sin ^{2}\left[\theta(d)-\theta_{p}\right] \\
& +\frac{1}{2} K_{13} \sin [2 \theta(d)] \theta^{\prime}(d)-\frac{1}{2} K_{13} \sin [2 \theta(0)] \theta^{\prime}(0)
\end{aligned}
$$

and the Euler-Lagrange equation is

$$
K \theta^{\prime \prime}+\frac{\Delta \chi B^{2}}{\mu_{0}} \sin \theta \cos \theta=0 .
$$

The various solutions are characterized by two arbitrary integration constants, as the Euler-Lagrange equation is a second-order ordinary differential equation. Both boundaries involve two boundary conditions, adding to a total of four boundary conditions:

$$
\frac{1}{2} C \sin \left\{2\left[\theta(d)-\theta_{p}\right]\right\}+\left\{K+K_{13} \cos [2 \theta(d)]\right\} \theta^{\prime}(d)=0,
$$

$$
\frac{1}{2} C \sin \left\{2\left[\theta(0)-\theta_{p}\right]\right\}-\left\{K+K_{13} \cos [2 \theta(0)]\right\} \theta^{\prime}(0)=0
$$

$$
\begin{aligned}
& \frac{1}{2} K_{13} \sin [2 \theta(d)]=0, \\
& \frac{1}{2} K_{13} \sin [2 \theta(0)]=0 .
\end{aligned}
$$

These four boundary conditions originate from the four degrees of freedom of the surface free energy, namely, $\theta(0), \theta(d), \theta^{\prime}(0)$, and $\theta^{\prime}(d)$. However, the Euler-Lagrange equation only allows for two degrees of freedom, because of the two arbitrary integration constants. In general a solution of the Euler-Lagrange equation that satisfies all four boundary conditions does not exist.

Apparently, the director field that minimizes the free energy cannot be found by variational calculus. It can even be shown that the free energy has no minimum. To that end a particular director field is considered, namely,

$$
\theta_{n}(x)=\theta_{d}+\beta\left[\left(\frac{x}{d}\right)^{n}-1\right]
$$

where $n$ is a large positive integer. The Frank free energy of the field is given by

$$
\begin{aligned}
\int_{0}^{d} d x \frac{1}{2} K \theta_{n}^{\prime 2} & =\frac{1}{2} \frac{K \beta^{2} n^{2}}{d^{2}} \int_{0}^{d} d x\left(\frac{x}{d}\right)^{2 n-2} \\
& =\frac{1}{2} \frac{K \beta^{2}}{d^{2}} \frac{n^{2}}{2 n-1} \\
& \approx \frac{1}{4} \frac{K \beta^{2}}{d^{2}} n .
\end{aligned}
$$

The elastic contribution of the splay-bend term reads

$$
\begin{array}{r}
\frac{1}{2} K_{13}\left\{\sin \left[2 \theta_{n}(d)\right] \theta_{n}^{\prime}(d)-\sin \left[2 \theta_{n}(0)\right] \theta_{n}^{\prime}(0)\right\} \\
\quad \approx \frac{K_{13} \sin \left(2 \theta_{d}\right) \beta}{2 d} n .
\end{array}
$$

The magnetic and anchoring energy remain finite for all $n$. Clearly, the free energy for large $n$ is given by

$$
F_{n}=\frac{1}{4} \frac{K \beta^{2}}{d^{2}}\left[1+\frac{2 K_{13} \sin \left(2 \theta_{d}\right)}{K \beta}\right] n .
$$

Choosing $\beta$ such that

$$
K_{13} \sin \left(2 \theta_{d}\right) \beta<0,
$$




$$
|\beta|<\frac{2\left|K_{13} \sin \left(2 \theta_{d}\right)\right|}{K}
$$

it follows that

$$
\lim _{n \rightarrow \infty} F_{n}=-\infty
$$

In the limit $n \rightarrow \infty$ it also holds that

$$
\lim _{n \rightarrow \infty} \theta_{n}(x)= \begin{cases}\theta_{d}-\beta & \text { if } 0 \leq x<d \\ \theta_{d} & \text { if } x=d .\end{cases}
$$

Clearly, the director field with a free energy diverging to minus infinity has a discontinuity at $x=d$. This discontinuity is referred to as the Oldano-Barbero boundary discontinuity.

The OB discontinuity also appears in the general case where the Euler-Lagrange equations are a pair of coupled second-order partial differential equations. The boundary discontinuity is attended by a free energy that diverges to minus infinity. This divergence is caused by the dependence of the surface term $f_{s \perp}$ on the derivative of the director field normal to the surface [6].

The OB paradox boils down to the appearance of a discontinuity in a theory that is based upon the absence of large deformations. Evidently the elastic free energy cannot be approximated by the five terms containing two spatial derivatives of the director field. The second criterion for the validity of the approximation is not met, i.e., there is no lower bound of the free energy. Finally, we mention that a variety of different ways of analyzing the OB paradox can be found in [11].

\section{THE FAETTI APPROACH}

Faetti's approach for solving the OB paradox is in the line of Barbero et al. [2,3]. These last authors solved the OB paradox by introducing one single higher-order elastic term, namely, a term quadratic in the secondorder derivatives of the director field. Then the result- ing Euler-Lagrange equations are differential equations of fourth order. Consequently four arbitrary integration constants specify the different solutions of the EulerLagrange equation relating to the splay-bend geometry considered in Sec. III. Now the variational problem has a well-defined solution. It should be remarked that the choice of the particular higher-order elastic term is ad hoc. Moreover, the combined action of the $K_{13}$ term and the term introduced by Barbero et al. gives rise to a large variation of the director field in a microscopically thin region near the boundary. Such a large variation of the director field in a microscopic region near the boundary is called a strong subsurface deformation.

According to Faetti [4] more higher-order terms must be introduced in the approach of Barbero et al. $[2,3]$. For the splay-bend geometry Faetti also finds a strong subsurface deformation of the director field. Following Barbero et al. [12] he states that the macroscopic effect of this deformation cannot be distinguished from the effect of anchoring forces. In a subsequent paper Faetti [5] generalized the approach to arbitrary geometries. According to Faetti the effect of the surface term $f_{s \perp}$ and of the higher-order terms is a strong subsurface deformation in any geometry. The only observable macroscopic effect of this strong subsurface deformation can be seen as an additional contribution to the anchoring energy. Denoting the sum of $f_{s \perp}$ and the higher-order terms by $\tau_{F}$ an effective anchoring function can be defined by

$$
\sigma^{\mathrm{eff}}=\sigma+\tau_{F}
$$

This effective anchoring energy $\sigma^{\text {eff }}$ can be approximated after Rapini and Papoular [9] as

$$
\sigma^{\mathrm{eff}}=-\frac{1}{2} C^{\mathrm{eff}}\left(\boldsymbol{\Pi}^{\mathrm{eff}} \cdot \boldsymbol{n}\right)^{2},
$$

where $\Pi^{\text {eff }}$ is the effective easy axis and $C^{\text {eff }}$ the effective anchoring constant. Now the variation of the free energy can be expressed as

$$
\begin{aligned}
\delta F= & \sum_{\chi=\theta, \phi} \int d V\left[\frac{\partial f_{m}}{\partial \chi}+\frac{\partial f_{b}}{\partial \chi}-\partial_{\beta}\left(\frac{\partial f_{b}}{\partial \partial_{\beta} \chi}\right)\right] \delta \chi \\
& +\sum_{\chi=\theta, \phi} \int d S\left\{\frac{\partial \sigma^{\mathrm{eff}}}{\partial \chi}+p_{\beta} \frac{\partial f_{b}}{\partial \partial_{\beta} \chi}+\frac{\partial f_{s \|}}{\partial \chi}-\sum_{i=1,2} \frac{1}{J\left(q_{1}, q_{2}\right)} t_{i \mu} \partial_{\mu}\left[J\left(q_{1}, q_{2}\right) t_{i \beta} \frac{\partial f_{s \|}}{\partial \partial_{\beta} \chi}\right]\right\} \delta \chi .
\end{aligned}
$$

Consequently the Faetti boundary conditions read

$$
\begin{aligned}
\frac{\partial \sigma^{\mathrm{eff}}}{\partial \chi} & +p_{\beta} \frac{\partial f_{b}}{\partial \partial_{\beta} \chi}+\frac{\partial f_{s \|}}{\partial \chi} \\
& -\sum_{i=1,2} \frac{1}{J\left(q_{1}, q_{2}\right)} q_{i \mu} \partial_{\mu}\left[J\left(q_{1}, q_{2}\right) q_{i \beta} \frac{\partial f_{s \|}}{\partial \partial_{\beta} \chi}\right]=0
\end{aligned}
$$

for $\chi=\theta, \phi$.

It should be emphasized here that Faetti's approach does not distinguish clearly between microscopy and macroscopy. Namely, a macroscopic theory, the continuum theory, describes a microscopic phenomenon, the strong subsurface deformation. Thereupon this microscopic deformation is interpreted macroscopically in terms of a contribution to the anchoring energy. In other 
words, the strong subsurface deformation is not consistent with the first condition for the validity of the approximation of the elastic free energy, stating that deformations must be small.

\section{THE PERGAMENSHCHIK APPROACH}

Pergamenshchik [6] assumed that the higher-order elastic terms are indeed extremely important but that their effect can be taken into account in terms of a constraint on the director field at the boundaries. Consequently these higher-order terms do not appear explicitly in the bulk equilibrium equations and the boundary conditions. So the equations for the bulk are the original Euler-Lagrange equations (28). Pergamenshchik's approach boils down to a constraint on the normal derivatives of the surface tilt and twist angles appearing in the surface term $f_{s \perp}$. These normal derivatives can no longer be considered as independent variables. Instead they depend on the surface tilt angle $\theta^{s}\left(q_{1}, q_{2}\right)$ and the surface twist angle $\phi^{s}\left(q_{1}, q_{2}\right)$.

According to Pergamenshchik the functional dependence of $f_{s \perp}$ on the surface tilt and twist angles can be found by solving the Euler-Lagrange equations (28) in terms of $\theta^{s}\left(q_{1}, q_{2}\right)$ and $\phi^{s}\left(q_{1}, q_{2}\right)$, where these surface angles are taken as fixed boundary conditions. Next the resulting expression of the director field is used to calculate the normal derivatives of the surface angles in order to determine $f_{s \perp}$. In general the dependence of $f_{s \perp}$ on the surface angles is nonlocal and cannot be given explicitly. In the following the proposal of Pergamenshchik for $f_{s \perp}$ will be denoted as

$$
f_{s \perp}=\tau\left(\left\{\theta^{s}\left(q_{1}, q_{2}\right), \phi^{s}\left(q_{1}, q_{2}\right)\right\}\right) .
$$

The still unknown surface angles are determined by minimizing the total free energy with respect to $\theta^{s}\left(q_{1}, q_{2}\right)$ and $\phi^{s}\left(q_{1}, q_{2}\right)$. This minimization requirement can be expressed in the form of boundary conditions. The minimization procedure results in

$$
\begin{aligned}
\frac{\delta F}{\delta \chi^{s}}= & \sum_{\chi=\theta, \phi} \int d V\left[\frac{\partial f_{m}}{\partial \chi}+\frac{\partial f_{b}}{\partial \chi}-\partial_{\beta}\left(\frac{\partial f_{b}}{\partial \partial_{\beta} \chi}\right)\right] \frac{\delta \chi}{\delta \chi^{s}} \\
& +\sum_{\chi=\theta, \phi} \int d S\left\{\frac{\partial \sigma}{\partial \chi}+p_{\beta} \frac{\partial f_{b}}{\partial \partial_{\beta} \chi}+\frac{\partial f_{s \|}}{\partial \chi}+\frac{\delta \tau}{\delta \chi^{s}}\right. \\
& \left.-\sum_{i=1,2} \frac{1}{J\left(q_{1}, q_{2}\right)} t_{i \mu} \partial_{\mu}\left[J\left(q_{1}, q_{2}\right) t_{i \beta} \frac{\partial f_{s \|}}{\partial \partial_{\beta} \chi}\right]\right\} \\
= & 0
\end{aligned}
$$

Using the Euler-Lagrange equations (28) the Pergamenshchik boundary conditions can be expressed as

$$
\begin{aligned}
\frac{\partial \sigma}{\partial \chi} & +p_{\beta} \frac{\partial f_{b}}{\partial \partial_{\beta} \chi}+\frac{\partial f_{s \|}}{\partial \chi}+\frac{\delta \tau}{\delta \chi^{s}} \\
& -\sum_{i=1,2} \frac{1}{J\left(q_{1}, q_{2}\right)} q_{i \mu} \partial_{\mu}\left[J\left(q_{1}, q_{2}\right) q_{i \beta} \frac{\partial f_{s \|}}{\partial \partial_{\beta} \chi}\right]=0
\end{aligned}
$$

for $\chi=\theta, \phi$. It should be remarked that a similar boundary condition was first postulated by Hinov [13].

According to Pergamenshchik the solution of the Euler-Lagrange equations (28) which minimizes the approximation of the free energy (16) gives rise to a minimum of the true free energy. This assumption cannot be tested directly, as explicit expressions for the higher-order elastic terms are unknown. However, an indirect test is quite possible by examining whether the Pergamenshchik approach is in agreement with the requirement for mechanical equilibrium. If this condition is not satisfied the proposed director field does not minimize the free energy. This test is originally due to Faetti [4]. A general formulation of the test is derived in the following.

A deformed nematic can only sustain its deformation if external forces and torques are applied to the nematic. In equilibrium, the forces and torques due to the elastic deformation are balanced at each material point by the external forces and torques. There are two sources of external torques.

The first source is the externally applied magnetic field. This field exerts a local torque density

$$
\begin{aligned}
\boldsymbol{M}^{\mathrm{mag}} & =-\varepsilon_{\alpha \beta \gamma} n_{\boldsymbol{\beta}} \frac{\partial f_{m}}{\partial n_{\gamma}} \hat{\boldsymbol{e}}_{\boldsymbol{\alpha}} \\
& =\frac{\Delta \chi}{\mu_{0}}(\boldsymbol{n} \cdot \boldsymbol{B})(\boldsymbol{n} \times \boldsymbol{B})
\end{aligned}
$$

on the nematic. This magnetic torque density is perpendicular to the plane spanned by the magnetic field and the director, i.e., it tends to align the director along the magnetic field. In case $\Delta \chi<0$ the tendency will be to align the director perpendicular to the magnetic field. The magnetic torque density can also be expressed in terms of the tilt angle $\theta$ and the twist angle $\phi$ as

$$
\boldsymbol{M}^{\mathrm{mag}}=\frac{\partial f_{m}}{\partial \theta}\left(\hat{\boldsymbol{e}}_{\boldsymbol{\theta}} \times \boldsymbol{n}\right)+\frac{1}{\sin \theta} \frac{\partial f_{m}}{\partial \phi}\left(\hat{\boldsymbol{e}}_{\boldsymbol{\phi}} \times \boldsymbol{n}\right),
$$

where $\hat{\boldsymbol{e}}_{\boldsymbol{\theta}}$ and $\hat{\boldsymbol{e}}_{\phi}$ are two orthonormal vectors perpendicular to $\boldsymbol{n}$ and defined by

$$
\begin{aligned}
& \hat{\boldsymbol{e}}_{\theta}=\frac{\partial \boldsymbol{n}}{\partial \theta}=(\cos \theta \cos \phi, \cos \theta \sin \phi,-\sin \theta), \\
& \hat{\boldsymbol{e}}_{\phi}=\frac{1}{\sin \theta} \frac{\partial \boldsymbol{n}}{\partial \phi}=(-\sin \phi, \cos \phi, 0) .
\end{aligned}
$$

The second source is the body on which the nematic is anchored. A mechanical surface force density $f^{\text {mech }}$ gives rise to a mechanical surface torque density

$$
M^{\mathrm{mech}}=\boldsymbol{r} \times \boldsymbol{f}^{\mathrm{mech}}
$$

whereas the anchoring gives rise to an anchoring surface torque density

$$
\begin{aligned}
\boldsymbol{M}^{\text {anch }} & =-\varepsilon_{\alpha \beta \gamma} n_{\beta} \frac{\partial \sigma}{\partial n_{\gamma}} \hat{\boldsymbol{e}}_{\boldsymbol{\alpha}} \\
& =C(\boldsymbol{n} \cdot \boldsymbol{\Pi})(\boldsymbol{n} \times \boldsymbol{\Pi}),
\end{aligned}
$$

or in terms of the tilt angle $\theta$ and the twist angle $\phi$ 


$$
\boldsymbol{M}^{\mathrm{anch}}=\frac{\partial \sigma}{\partial \theta}\left(\hat{\boldsymbol{e}}_{\boldsymbol{\theta}} \times \boldsymbol{n}\right)+\frac{1}{\sin \theta} \frac{\partial \sigma}{\partial \phi}\left(\hat{\boldsymbol{e}}_{\boldsymbol{\phi}} \times \boldsymbol{n}\right) .
$$

The anchoring torque density (53) tends to align the surface director along the easy direction $\Pi$.

In equilibrium, the total externally applied force on the nematic and the total externally applied torque on the nematic must be zero. Consequently, the equations describing the mechanical equilibrium of the nematic are

$$
\begin{gathered}
\int d S f^{\mathrm{mech}}=\mathbf{0} \\
\int d S M^{\mathrm{mech}}+\int d S M^{\mathrm{anch}}+\int d V M^{\mathrm{mag}}=\mathbf{0} .
\end{gathered}
$$

Besides the torque balance equation (55b) referring to the nematic, similar equations must be formulated referring to the anchoring body. According to Newton's third law the anchoring body experiences a torque exerted by the nematic. This torque $\boldsymbol{M}^{\text {nem }}$ is equal in magnitude to the torque exerted by the anchoring body, but of opposite sign, i.e.,

$$
M^{\text {nem }}=-\int d S M^{\text {mech }}-\int d S M^{\text {anch }}
$$

Clearly this torque on the anchoring body must be balanced by another torque $\boldsymbol{M}^{\text {ext }}$ which is mechanically applied to the anchoring body:

$$
M^{\text {ext }}+M^{\text {nem }}=0 .
$$

Now the relevant equations for the Faetti test can be formed. These equations describe the equilibrium of torques of the combined system, i.e., the system consisting of the nematic and anchoring body. Using (55b),
(56), and (57) it follows that

$$
M^{\text {ext }}+\int d V M^{\mathrm{mag}}=0 .
$$

An expression for $\boldsymbol{M}^{\text {ext }}$ will be derived below in order to test the mechanical equilibrium.

To that end the combined system is subjected to a rotation with infinitesimal angle $\delta \omega$ around the axis parallel to the unit vector $\boldsymbol{k}$. Then the change in the total free energy is

$$
\delta F=M_{\alpha}^{\mathrm{ext}} \delta \omega_{\alpha},
$$

where $\delta \omega_{\alpha}=\delta \omega k_{\alpha}$. In order to derive an expression for $M_{\alpha}^{\text {ext }}$ it must be realized that a rotation of the combined system over an angle $\delta \omega$ around the axis $\boldsymbol{k}$ is equivalent to a rotation of the magnetic field over an angle $-\delta \omega$ around the axis $\boldsymbol{k}$. Then the change in the magnetic field is

$$
\delta B_{\alpha}=-\varepsilon_{\alpha \beta \gamma} \delta \omega_{\beta} B_{\gamma} .
$$

The corresponding change in the Eulerian angles parametrizing the director field can be expressed as

$$
\delta \chi=\frac{\partial \chi}{\partial B_{\beta}} \delta B_{\beta}
$$

where $\chi$ is the solution of the Euler-Lagrange equations (28) and the boundary conditions (48). Next the change in the total free energy is calculated in terms of $\delta \boldsymbol{B}$. For that purpose it must be realized that the free energy depends both explicitly and implicitly on $\boldsymbol{B}$. The explicit dependence is brought about by the contributions $f_{m}$ and $\tau$, for $\tau$ depends on the normal derivatives of the surface angles, which follow from the Euler-Lagrange equations. The implicit dependence is due to the Eulerian angles $\theta$ and $\phi$. Using Eqs. (28) and (48) it follows that

$$
\begin{aligned}
\delta F= & \int d V \frac{\partial f_{m}}{\partial B_{\alpha}} \delta B_{\alpha}+\int d S \frac{\partial \tau}{\partial B_{\alpha}} \delta B_{\alpha}+\sum_{\chi=\theta, \phi} \int d V\left[\frac{\partial f_{m}}{\partial \chi}+\frac{\partial f_{b}}{\partial \chi}-\partial_{\beta}\left(\frac{\partial f_{b}}{\partial \partial_{\beta} \chi}\right)\right] \frac{\partial \chi}{\partial B_{\alpha}} \delta B_{\alpha} \\
& +\sum_{\chi=\theta, \phi} \int d S\left\{\frac{\partial \sigma}{\partial \chi}+p_{\beta} \frac{\partial f_{b}}{\partial \partial_{\beta} \chi}+\frac{\partial f_{s \|}}{\partial \chi}-\sum_{i=1,2} \frac{1}{J\left(q_{1}, q_{2}\right)} t_{i \mu} \partial_{\mu}\left[J\left(q_{1}, q_{2}\right) t_{i \beta} \frac{\partial f_{s \|}}{\partial \partial_{\beta} \chi}\right]+\frac{\delta \tau}{\delta \chi^{s}}\right\} \frac{\partial \chi^{s}}{\partial B_{\alpha}} \delta B_{\alpha} \\
= & -\int d V \varepsilon_{\alpha \beta \gamma} B_{\beta} \frac{\partial f_{m}}{\partial B_{\gamma}} \delta \omega_{\alpha}-\int d S \varepsilon_{\alpha \beta \gamma} B_{\beta} \frac{\partial \tau}{\partial B_{\gamma}} \delta \omega_{\alpha} .
\end{aligned}
$$

The integrand of the first term on the right hand side can be rewritten using Eq. (13) for $f_{m}$ and Eq. (49) for $M^{\text {mag: }}$

$$
\begin{aligned}
\varepsilon_{\alpha \beta \gamma} B_{\beta} \frac{\partial f_{m}}{\partial B_{\gamma}} & =-\varepsilon_{\alpha \beta \gamma} n_{\beta} \frac{\partial f_{m}}{\partial n_{\gamma}} \\
& =M_{\alpha}^{\mathrm{mag}} .
\end{aligned}
$$

Then it follows:

$$
\delta F=-\int d V M_{\alpha}^{\mathrm{mag}} \delta \omega_{\alpha}-\int d S \varepsilon_{\alpha \beta \gamma} B_{\beta} \frac{\partial \tau}{\partial B_{\gamma}} \delta \omega_{\alpha}
$$

Using (59) it follows that $M_{\alpha}^{\text {ext }}$ satisfies

$$
M_{\alpha}^{\mathrm{ext}}+\int d V M_{\alpha}^{\mathrm{mag}}=-\int d S \varepsilon_{\alpha \beta \gamma} B_{\beta} \frac{\partial \tau}{\partial B_{\gamma}} .
$$

According to the Faetti test equilibrium of torques exists 
provided that

$$
\frac{\partial \tau}{\partial B_{\alpha}}=\lambda B_{\alpha}
$$

where $\lambda$ is an arbitrary number. Equation (66) implies that $\tau$ only depends on the magnitude of $\boldsymbol{B}$, i.e., $\tau$ only depends on $\boldsymbol{B}^{2}$.

According to Pergamenshchik the contribution $\tau$ to the surface free energy does depend on the direction of the magnetic field as well. The reason is the constraint satisfied by the normal derivatives of the surface angles. Therefore his approach does not give rise to a director field that minimizes the free energy.

It should be remarked here that Faetti's approach does not violate the requirement for mechanical equilibrium. His contribution to the surface free energy $\tau_{F}$, which corresponds to the quantity $\tau$ of Pergamenshchik, is a local function of the director field only. The independence of $\tau_{F}$ on the magnetic field implies directly that Eq. (66) is satisfied.

\section{DISCUSSION}

In the preceding sections it is shown that none of the existing approaches is completely satisfying. The essential element in the approach of Barbero et al. [2,3] and of Faetti $[4,5]$ is the strong subsurface deformation, that is not consistent with the requirement of small deformations. The approach of Pergamenshchik [6] does not give rise to mechanical equilibrium, i.e., the proposed director field does not lead to the minimum of the free energy. Despite these imperfections successes have been achieved in describing some experimental results. The approach of Barbero et al. seems to be useful in the description of so-called anchoring transitions $[14,15]$. Anchoring transitions involve changes in the easy axis with variations in temperature. The variation of this preferential direction as a function of temperature is nonanalytical at the anchoring transition temperature. The approach of Pergamenshchik, on the other hand, seems to be useful in the description of instabilities in thin hybrid aligned nematic liquid-crystal films [16]. However, these experiments cannot be considered conclusive evidence for either approach.

The approach of Faetti may be reformulated without any reference to strong subsurface deformations. This reformulated approach constitutes a solution of the OB paradox which is in agreement with both conditions for the validity of the approximated elastic free energy. The basic assumption is that the bulk elastic free energy can be approximated according to Frank [7]. Then the equilibrium equations for the bulk are the Euler-Lagrange equations (28). Accordingly, strong anchoring can be seen as the limiting case of an infinite anchoring constant, as the anchoring constant only enters the boundary conditions and not the equilibrium equations for the bulk. The inclusion of higher-order elastic terms, as in the theory of Barbero et al. [2,3], would result in strong subsurface deformations. This is prohibited by the first condition on the validity of the approximated elastic free energy, stating that deformations must be small.

In order to arrive at a well-defined solution of the OB paradox we must require that the number of degrees of freedom in the surface free energy must correspond to the order of the differential equations for the bulk. For example, consider the case where the EulerLagrange equations are second-order ordinary differential equations. Then the number of surface degrees of freedom must be equal to the number of arbitrary integration constants appearing in the general solution of the EulerLagrange equations. In the present case the number of boundary conditions (29) is not appropriate, as the bulk equations (28) are differential equations of second order. In order to obtain the correct number of boundary conditions an additional constraint on the surface free energy density is needed. The superfluous boundary conditions can be removed by assuming that the surface elastic free energy density does not depend on the derivative of the director field normal to the surface. This means that the surface free energy density $f_{s \perp}$ must be excluded. Now the resulting free energy can be minimized using variational calculus, without being confronted with the $O B$ paradox. The inclusion of a term in the surface free energy density, which is linear in the normal derivatives of the director field, leads to a free energy that is unbounded from below. This is forbidden by the second condition on the validity of the approximated elastic free energy, namely, the condition that the approximated elastic free energy must have a minimum. Taking into account the surface term $f_{s \perp}$ in another way, as in the approach of Pergamenshchik [6], results in a violation of the requirement of mechanical equilibrium. It should be noted that our proposal is not just to set $f_{s \perp}$ equal to zero, but instead not allowing this term to appear in the surface free energy density, for the reasons discussed above.

Summarizing, the present proposal is to approximate the elastic free energy by

$$
\begin{aligned}
F_{\mathrm{el}}= & \int d V f_{b}+\int d S f_{s \|} \\
= & \int d V\left\{\frac{1}{2} K_{11}(\boldsymbol{\nabla} \cdot \boldsymbol{n})^{2}+\frac{1}{2} K_{22}[\boldsymbol{n} \cdot(\boldsymbol{\nabla} \times \boldsymbol{n})]^{2}+\frac{1}{2} K_{33}[\boldsymbol{n} \times(\boldsymbol{\nabla} \times \boldsymbol{n})]^{2}\right\} \\
& +\int d S\left\{\frac{1}{2}\left(K_{22}+K_{24}\right) \boldsymbol{p} \cdot[(\boldsymbol{n} \cdot \boldsymbol{\nabla}) \boldsymbol{n}-\boldsymbol{n}(\boldsymbol{\nabla} \cdot \boldsymbol{n})]+K_{13}(\boldsymbol{p} \cdot \boldsymbol{n})[\boldsymbol{\nabla} \cdot \boldsymbol{n}-\boldsymbol{p} \cdot(\boldsymbol{p} \cdot \nabla) \boldsymbol{n}]\right\}
\end{aligned}
$$


Then the equilibrium equations for the bulk are the Euler-Lagrange equations

$$
\partial_{\beta}\left(\frac{\partial f_{b}}{\partial \partial_{\beta} \chi}\right)-\frac{\partial f_{b}}{\partial \chi}-\frac{\partial f_{m}}{\partial \chi}=0
$$

for $\chi=\theta, \phi$, whereas the boundary conditions are

$$
\begin{aligned}
\frac{\partial \sigma}{\partial \chi} & +p_{\beta} \frac{\partial f_{b}}{\partial \partial_{\beta} \chi}+\frac{\partial f_{s \|}}{\partial \chi} \\
& -\sum_{i=1,2} \frac{1}{J\left(q_{1}, q_{2}\right)} q_{i \mu} \partial_{\mu}\left[J\left(q_{1}, q_{2}\right) q_{i \beta} \frac{\partial f_{s \|}}{\partial \partial_{\beta} \chi}\right]=0
\end{aligned}
$$

for $\chi=\theta, \phi$.

\section{CONCLUSION}

After a discussion of the OB paradox the existing approaches for solving this paradox are analyzed in view of two starting points of the continuum theory. Namely, the deformations of the director field must be small and the approximated free energy must have a minimum. The present analysis clearly shows the imperfections of the approaches of Barbero et al., Faetti, and Pergamenshchik. A reformulation of Faetti's approach is proposed which is in agreement with the assumptions of the continuum theory. The proposal boils down to a modification of the original form of the surface free energy density, namely, the derivatives of the director field normal to the surface must be excluded.

\section{ACKNOWLEDGMENT}

We wish to thank J.A.M.M. van Haaren of the Philips Research Laboratories for stimulating discussions.
[1] C. Oldano and G. Barbero, J. Phys. (Paris) Lett. 46, 451 (1985); Phys. Lett. 110A, 213 (1985); G. Barbero and C. Oldano, Nuovo Cimento D 6, 479 (1985).

[2] G. Barbero, N. V. Madhusudana, and C. Oldano, J. Phys. (Paris) 50, 2263 (1989).

[3] G. Barbero, A. Sparavigna, and A. Strigazzi, Nuovo Cimento D 12, 1259 (1990).

[4] S. Faetti, Phys. Rev. E 49, 5332 (1994).

[5] S. Faetti, Phys. Rev. E 49, 4192 (1994).

[6] V. M. Pergamenshchik, Phys. Rev. E 48, 1254 (1993).

[7] F. C. Frank, Discuss. Faraday Soc. 25, 19 (1958).

[8] J. Nehring and A. Saupe, J. Chem. Phys. 54, 337 (1971).

[9] A. Rapini and M. Papoular, J. Phys. (Paris) Colloq. 30,
C4-54 (1969).

[10] I. Dahl and A. De Meyere, Liq. Cryst. 18, 683 (1995).

[11] S. Ponti, Phys. Lett. A 200, 165 (1995).

[12] G. Barbero, Z. Gabbasova, and Yu. A. Kosevich, J. Phys. (France) II 1, 1505 (1991).

[13] H. P. Hinov, Mol. Cryst. Liq. Cryst. 148, 197 (1987).

[14] G. Barbero and G. Durand, Phys. Rev. E 48, 1942 (1993).

[15] T. Beica, S. Frunza, R. Moldovan, and S. Ponti, Phys. Lett. A 197, 164 (1995).

[16] O. D. Lavrentovich and V. M. Pergamenshchik, Phys. Rev. Lett. 73, 979 (1994). 